\title{
Interdisciplinary Learning
}

\section{Introduction}

Scottish education is going through a period of significant and important change to its curriculum, learning and teaching, and the processes that underpin them. Education Scotland is supporting this change by evaluating and sharing evolving practice as part of a national professional learning community. This is the fourth in a series of briefings which provide succinct advice on the progress being made in key areas of change, to help inform discussion and promote further, innovative development. This latest addition to the series explores interdisciplinary learning.
The curriculum should include space for learning beyond subject boundaries, so that children and young people can make connections between different areas of learning. Interdisciplinary studies, based upon groupings of experiences and outcomes from within and across curriculum areas, can provide relevant, challenging and enjoyable learning experiences and stimulating contexts to meet the varied needs of children and young people. Building the Curriculum 3 (BtC3). 


\section{Interdisciplinary learning is a planned approach to learning which uses links across different subjects or disciplines to enhance learning.}

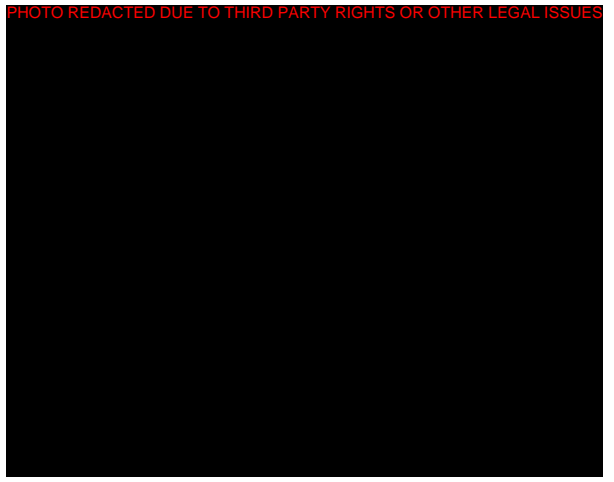

What is interdisciplinary learning?

Interdisciplinary learning, sometimes known as interdisciplinary studies, is a planned approach to learning which uses links across different subjects or disciplines to enhance learning. It promotes the development and application of what has been taught and learned in new and different ways. It provides opportunities for deepening learning, for example through exploring an issue, solving problems or completing a final project. It is an important approach at all levels of Curriculum for Excellence and can take place in curriculum areas and subjects, the ethos and life of the school and community, and a range of other opportunities for personal development. It is a particularly useful way of promoting learning across the broad general education, as well as giving coherence to learning at the senior phase. Usually, interdisciplinary learning can be recognised by one or more of the following characteristics.

- It may be individual one-off projects or longer courses of study.

- It is planned around clear purposes.

- It is based upon experiences and outcomes drawn from different curriculum areas or subjects within them.

- It ensures progression in skills and in knowledge and understanding.

- It can provide opportunities for mixed stage learning which is interest based.

\section{What does it look like?}

Two distinct and complementary approaches are emerging in effective practice.

- The first approach is characterised by learning which is planned to

\section{develop awareness and understanding of the connections and differences across subjects or curriculum}

areas. This may be achieved through the knowledge developed, or the ways of working, or the attributes, capabilities and skills (including higher-order thinking skills) being consolidated, or through a particular perspective given by different subjects. A good example is when practitioners find the common ground between two or more subjects so that they can use it to explore an idea in depth, such as when the teaching of probability in mathematics is co-ordinated with science work on DNA and Genetics. In this way, learning is more relevant because learners gain a better understanding of a concept by appreciating its application in the real world.

Revisiting a concept or skill from different perspectives deepens understanding and can also make the curriculum more coherent and meaningful from the learners' point of view. Interdisciplinary studies can also take advantage of opportunities to work with partners who are able to offer and support enriched learning experiences and opportunities for young people's wider involvement in society. BtC3

- The second approach is when learning in different subjects or curriculum areas is used to explore

a theme or an issue, meet a challenge, solve a problem or complete a final project. This can be achieved by providing a context that is real and relevant to the learners, the school and its community. This second approach is perhaps the more traditional, well-understood look of interdisciplinary learning. When most effective, the emphasis tends to be on the process of learning and exploration of the interaction between the subjects, not just on the context or theme. For example, to promote ICT skills and creativity, or indeed learning about Scotland, children might use knowledge and skills developed in the study of local history, art and design and French language to create informative and attractive web pages for their peers in a French school.

Other approaches to organising learning are also being used in schools but these do not necessarily provide the benefits of interdisciplinary learning.

These are described here.

- Themed days or focus weeks are sometimes offered but not as part of an overall framework, or they may not have been planned in terms of learners' next steps and overall progression within curriculum areas. However, whilst these contexts for learning might be useful, they do need to be carefully planned to ensure learners progress and achieve well in the medium and long term.

- In cases where there are few common features in thematic work across subjects, specific experiences and outcomes (Es and Os) need to become the starting point and carefully planned. 


\section{This means looking closely at progression over time in knowledge, attributes and capabilities, and skills (including higher-order thinking skills)}

- Links in curriculum areas are sometimes chosen to fit a context but may not be based on progression and coherence in learning, sometimes because the links are tenuous. Such approaches need to be reviewed carefully against the opportunities presented by well-planned interdisciplinary learning.

\section{How is interdisciplinary learning planned?}

Interdisciplinary learning is most effective when it is tailored to and meets learners' needs. To do this, practitioners might combine curriculum areas within a theme or context and at the same time ensure that the learning is based on clearly identified next steps. This means looking closely at progression over time in knowledge, attributes and capabilities, and skills (including higher-order thinking skills). Sometimes, too much focus is given to fitting curriculum areas to the theme and important next steps in learning are neglected as a result. Practitioners are finding it particularly beneficial to involve learners fully in planning,

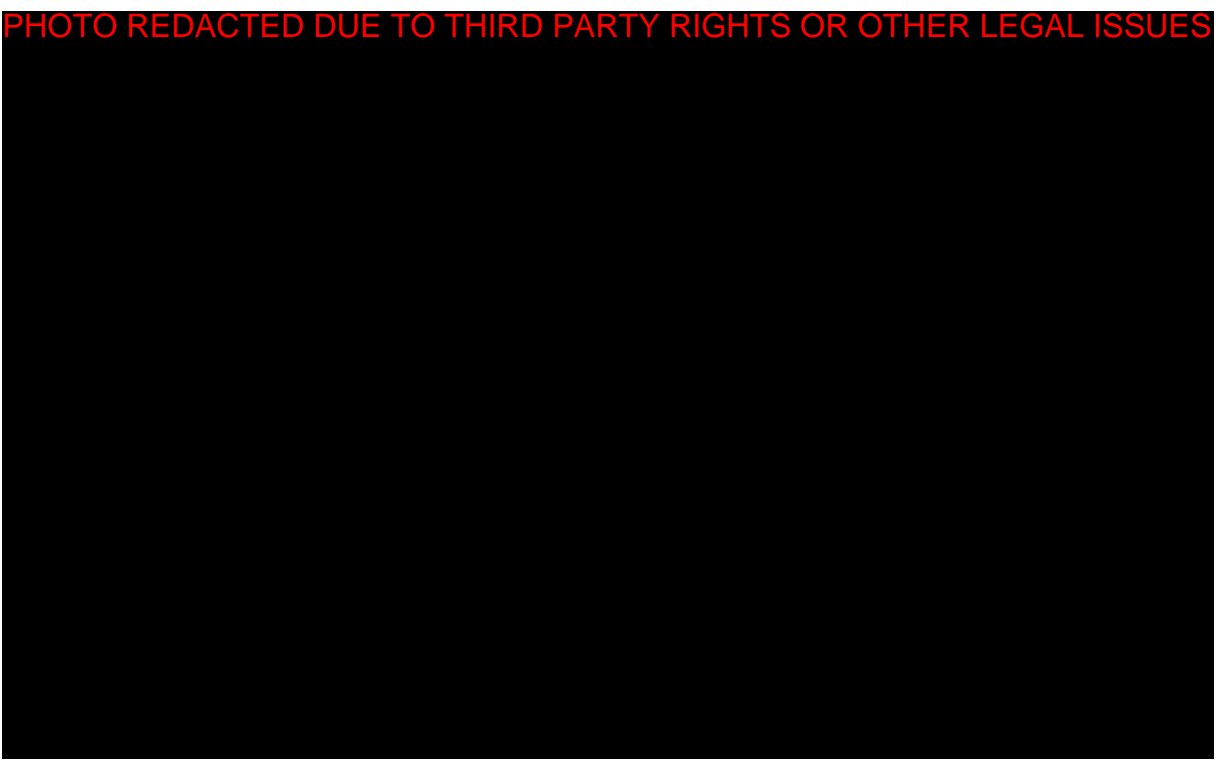

identifying their own next steps and building these into the plan from the outset. Whilst learners might also be involved in choosing a theme or a topic, unless they are fully involved in detailed content planning they are likely to miss out on important opportunities to build on their previous learning. In effective practice, staff have found it helpful to have a clear reason for selecting Es and Os from a few areas of the curriculum and then to use these to deliver one of the two approaches to interdisciplinary learning described above. Other features of effective planning for interdisciplinary learning include:

- starting with a few carefully selected Es and Os;

- having a clear purpose for using one or other of the two approaches to interdisciplinary learning;

- involving learners in planning by building on their existing knowledge and skills, whilst also ensuring progression and coherence within an overall framework which has been designed by staff based on the principles of curriculum design;

- ensuring that learners and staff are clear about the knowledge and skills

\section{being developed through}

interdisciplinary learning, and that this does not become lost within the context. Reflection sessions at the end of lessons can help staff and learners to discuss which areas of the curriculum were combined and what knowledge and skills from these curriculum areas were applied and deepened through the learning; and

- identifying clear learning intentions and success criteria within shortterm planning.

Effective interdisciplinary learning is often based around a few big ideas which describe the interdisciplinary elements of combining a few curriculum areas. In other words, by combining content from two or more curriculum areas, being really clear about what we want children and young people to learn. This often involves:

- identifying the interdisciplinary elements within the selected Es and Os;

- creating interdisciplinary learning outcomes;

- sharing the interdisciplinary learning outcomes/big ideas with children and young people; and

- using the interdisciplinary learning outcomes or big ideas as the main focus for assessment.

When considering long-term planning for progression, many schools and clusters of schools are developing a framework for interdisciplinary learning to ensure that learners experience their entitlement to a broad general education. This can help to ensure that interdisciplinary learning is planned around learners' needs and involves different curriculum areas as appropriate. This approach also helps 


\section{Effective interdisciplinary learning is often based around a few big ideas which describe the interdisciplinary elements of combining a few curriculum areas}

to ensure that children and young people build on their previous learning and reduces the risk of repetition at different stages.

Staff are using successfully the same principles in approaching interdisciplinary learning as they do in approaching other forms of learning. In best practice, interdisciplinary learning provides a

\section{stimulating and self-motivating}

context for learning and is both

enjoyable and relevant. It leads to a better, more rounded understanding of important ideas and to an increased competence in using knowledge and skills in transferable ways. Staff will be clear about the connections across learning that they want children and young people to explore and understand. They will also know what children and young people have learned previously and how they will apply and develop

this learning in new and different ways. Everyone involved will know which skills and ideas from different subjects or disciplines they are bringing together, and why.

\section{How can we take forward interdisciplinary learning?}

Interdisciplinary learning is most successful when it is underpinned by

effective self-evaluation which includes everyone involved. This means strong teamwork and effective dialogue amongst practitioners with the full involvement of learners and, as appropriate, their parents. It also means learning from elsewhere where others might be developing similar approaches, all with a clear and unobstructed focus on the needs of learners and their progress in learning. This means ensuring rigour in approaches to monitoring and tracking progress to ensure that learning is clearly focused on next steps. The potential benefits for learners who participate fully in well-conceived interdisciplinary learning experiences are becoming recognised. As you explore these benefits for learners, you may find it helpful to consider together with colleagues the following points.

- Are you clear about why you are using interdisciplinary learning, in preference to other forms of learning, to deliver the intended learning outcomes?

- How will you ensure that the interdisciplinary learning experience has progressed learners' knowledge, attributes, capabilities and skills, including higher-order thinking skills?

- How well does your project or course of study develop awareness and understanding of the connections and differences across subject areas and disciplines?

- How effectively does the learning explore a theme or an issue, meet a challenge or ensure a final product?

- To what extent does planning reflect the principles of curriculum design and build on children and young people's prior learning?

- Are learners given appropriate responsibility for and choice in planning both the learning, and perhaps the topic, within the context of an overall framework?

- Have you considered how to select and connect relevant Es and Os, and to maintain focus by not using too many?

- How clearly are interdisciplinary learning outcomes or big ideas for the project identified and shared with children and young people? Are these used as the basis for assessment?

- How effectively are opportunities to develop literacy, numeracy and health and wellbeing, included?

- Are learning intentions and success criteria clearly specified?

- Are you planning for appropriate learning opportunities outwith the classroom, including through the ethos and life of the school or other contexts for personal achievement?

- To what extent will you be working with parents, colleagues in the cluster, or partners in the community, both to ensure they understand what the benefits of interdisciplinary learning are and to enrich the experience for learners?

- Do you have a clear whole-school framework which ensures learners do not encounter the same topic or theme year after year without good reason?

- Where appropriate, are you using interdisciplinary learning effectively to enhance Gaelic medium provision?

\section{The CfE Briefings is a new series designed to provide practitioners with information and advice}

to support their implementation of $\mathrm{CfE}$. 